\title{
Clinical relevance of Cyr61 expression in patients with hormone-dependent breast cancer
}

\author{
SEBASTIAN MAYER ${ }^{1,2}$, THALIA ERBES ${ }^{1,2}$, SYLVIA TIMME-BRONSERT $^{2,3}$, MARKUS JAEGER ${ }^{1,2}$, \\ GERTA RÜCKER $^{2,4}$, FRANCISKA KUF ${ }^{1,2}$, ELMAR STICKELER $^{5}$, GERALD GITSCH $^{1,2}$ and MARC HIRSCHFELD ${ }^{1,2,6}$ \\ ${ }^{1}$ Department of Obstetrics and Gynecology, Medical Center - University of Freiburg; ${ }^{2}$ Faculty of Medicine, University of \\ Freiburg; ${ }^{3}$ Institute of Surgical Pathology, Department of Pathology, ${ }^{4}$ Institute for Medical Biometry and Statistics, Medical \\ Center - University of Freiburg, D-79106 Freiburg; ${ }^{5}$ Department of Gynecology and Obstetrics, University Medical Center \\ RWTH Aachen, D-52062 Aachen; ${ }^{6}$ Institute of Veterinary Medicine, University of Göttingen, D-37073 Göttingen, Germany
}

Received November 28, 2016; Accepted April 7, 2017

\section{DOI: $10.3892 / \mathrm{ol} .2017 .6406$}

\begin{abstract}
Tumor resistance to endocrine therapy triggers estrogen-independent cancer progression, which is a major obstacle to the successful treatment of hormone receptor positive breast cancer (BC). The underlying molecular mechanisms of endocrine resistance are not fully understood yet. The matricellular protein cysteine-rich angiogenic inducer 61 (Cyr61) is associated with tumor invasiveness and the induction of tumorigenesis in various malignancies in vivo and the induction of estrogen-independence and endocrine therapy resistance in $\mathrm{BC}$. The present study evaluated the potential effects and clinical relevance of Cyr61 expression levels in 67 patients with primary non-metastatic BC. Immunohistochemical analysis of formalin-fixed paraffin-embedded tissue sections was performed, and the association between Cyr61 protein expression and clinicopathological factors and survival was analyzed. Cyr61 overexpression was revealed to be significantly associated with a positive estrogen receptor (ER)/progesterone receptor $(\mathrm{PR})$ status $(\mathrm{P}=0.016)$ and to the molecular subtype of $\mathrm{BC}(\mathrm{P}=0.039)$. Compared with patients without Cyr61 overexpression, patients with Cyr61 overexpression exhibited an increased recurrence rate (30.6 vs. 22.6\%) and decreased long-term survival (10-year overall survival, 62.9 vs. $69.7 \%$ ); however, these associations did not reach statistically significant levels in Cox regression model analysis. Similar results were identified in the subgroup analysis of patients with ER/PR positive BC. These results indicate that Cyr61 serves a role in the development of endocrine therapy resistance in $\mathrm{BC}$ and is thus a potential therapeutic target to overcome endocrine therapy resistance. However, additional
\end{abstract}

Correspondence to: Dr Sebastian Mayer, Department of Obstetrics and Gynecology, Medical Center - University of Freiburg, 55 Hugstetter Street, D-79106 Freiburg, Germany

E-mail: sebastian.mayer@uniklinik-freiburg.de

Key words: cysteine-rich angiogenic inducer 61, breast cancer, endocrine resistance, overall survival, luminal like long-term survival analyses with large patient populations are required.

\section{Introduction}

Estrogen receptor (ER) and/or progesterone receptor (PR) expression characterizes $\sim 70 \%$ of all cases of breast cancer (BC) $(1,2)$. Thus, the majority of patients with BC are affected by the estrogen signaling pathway, which serves an important role in the development and progression of BC $(1,2)$. Therefore, endocrine therapy is an important treatment strategy for all stages of hormone-dependent BC. Various therapeutic agents, including selective ER modulators, gonadotropin-releasing hormone agonists and aromatase inhibitors, are used in the endocrine therapy of hormone-dependent BC and have demonstrated substantial clinical benefits, including improved disease control and survival outcomes (3). However, not all patients with hormone-receptor positive $\mathrm{BC}$ respond to endocrine therapy, termed de novo-resistance, and a substantial number of patients who initially respond to endocrine therapy later suffer from recurrence or disease progression, termed acquired resistance (2). Potential reasons for endocrine resistance include the following: Loss or modification of ER $\alpha$ expression; ER $\alpha$ mutation; the altered regulation of signaling pathways (phosphoinositide 3-kinase/protein kinase B/mechanistic target of rapamycin and cyclin-dependent kinase 4/6 signaling pathways); cross talk between the ER and growth factor receptor signaling pathways [human epidermal growth factor receptor 2 (HER2)]; altered expression of specific microRNAs; and interactions between the tumor microenvironment and host immune response. Although significant progress has been made in understanding the underlying molecular mechanisms of ligand-independent activation of the ER, the mechanisms of endocrine resistance remain unclear $(4,5)$.

The functional versatility of the matricellular protein cysteine-rich angiogenic inducer 61 (Cyr61) is recognized by a growing number of studies, which identified that Cyr61 serves a multitude of regulatory functions and has a range of potential binding partners, including integrins (6-9). Cyr61 is associated with essential signaling pathways in physiological processes, 
including angiogenesis $(7,10)$; however, the alteration of Cyr61 expression has been demonstrated to be associated with a number of pathologies (11-13), including malignant neoplasms $(14,15)$.

In previous studies by our group, Cyr61 expression profiling in $\mathrm{BC}$ and its functional expression regulation was examined $(16,17)$ demonstrating a stage-dependent induction of Cyr61 in BC tumorigenesis and hypoxia-induced alternative splicing of Cyr61 in tumor cells. The results of previous in vitro $(18,19)$ and in vivo $(20-23)$ investigations have highlighted the importance of Cyr61 in BC and the potential application of this knowledge for the management of cancer. In combination with Y-box-binding protein 1, Cyr61 acts on the urokinase plasminogen activator surface receptor to stimulate the progression of triple-negative BC (TNBC), while the expression of Cyr61 correlates with increased malignancy and poorer patient survival (20). In addition, high grade ductal carcinoma in situ may be characterized by Cyr61 expression, independent of the ER expression status, suggesting that Cyr61 serves a role in the development of intraepithelial carcinoma (23). In TNBC Cyr61 is described as a mediator of the proto-oncogene tyrosine-protein kinase Src signaling pathway, which modulates the metastatic potential of malignant cells (18).

The functional role of Cyr61 in overcoming ER dependency, which was suggested by Tsai et al (24), is of clinical importance. Using MCF7 cells and a mouse model, Tsai et al (24) demonstrated that Cyr61 was sufficient to induce estrogen independence and antiestrogen resistance. In addition, Jia et al (21) reported that patients with Cyr61-expressing hormone-dependent $\mathrm{BC}$ exhibited a poor response to letrozole treatment. The potential of Cyr61 as a therapeutic target in $\mathrm{BC}$ management was also demonstrated by another in vivo study (22).

The present study aimed to evaluate the potential effects and clinical relevance of Cyr61 expression in patients with primary non-metastatic BC. Therefore Cyr61 expression levels were correlated to tumor characteristics and survival data.

\section{Materials and methods}

Patients and treatment. Tumor specimens were obtained from 67 patients with histologically diagnosed primary non-metastatic invasive $\mathrm{BC}$ who received treatment at the Department of Obstetrics and Gynecology of the Medical Center of the University of Freiburg (Freiburg, Germany) between April 2000 and September 2001, and for whom Cyr61 measurement data was available. The present study was approved by the Ethics Committee of the University of Freiburg (approval no. 313/2001) and all patients provided written informed consent.

Clinicopathological characteristics of the patients, including age, histological tumor type, tumor grade, ER, PR and HER 2 expression statuses and molecular BC subtype were evaluated. The median age of the patients was 58 years at the time of diagnosis (range, 33-87 years). The characteristics of the study population are summarized in Table I.

The histological type of all tumors was categorized according to the World Health Organization Histological Typing of Breast Tumors (25). Tumor grade was defined according to Black's nuclear grading system with modification of numbers. ER, PR and HER2 expression statuses were determined by immunohistochemistry (IHC). Molecular BC subtypes were defined by IHC assessment as follows: Luminal-like (HER2 negative, ER/PR positive); HER2/luminal (HER2/ER/PR positive); HER2-like (HER2-positive, ER/PR negative); and triple-negative (HER2/ER/PR-negative).

All patients were treated according to national guidelines (26) with surgery (breast-conserving surgery/mastectomy with sentinel node biopsy and/or axillary dissection), which was followed by adjuvant chemotherapy, radiation and antihormonal therapy depending on the tumor stage and characteristics. Follow-up data [disease-free survival (DFS) and overall survival (OS)] were obtained from the local cancer registry, clinical records and from the general practitioners of the patients. Patients were censored at the time they were last observed alive.

$I H C$. Formalin-fixed paraffin-embedded BC tissue specimens, which were taken during surgery, were sectioned (3- $\mu$ m-thick) and stained for Cyr61, human transformer-2 protein homolog $\beta$ (hTRA2 $\beta$ ) and cluster of differentiation (CD) 44 proteins. After removing the paraffin with xylene and a descending series of alcohol, antigen retrieval was performed for $10 \mathrm{~min}$ in a microwave oven at $600 \mathrm{~W}$ using High $\mathrm{pH}$ Target Retrieval Solution (cat. no. K8004, Dako; Agilent Technologies GmbH, Waldbronn, Germany). Endogenous peroxidase activity was blocked using EnVision ${ }^{\mathrm{TM}}$ Flex Peroxidase Blocking Reagent (Dako; Agilent Technologies $\mathrm{GmbH}$ ) for $10 \mathrm{~min}$ at room temperature. The sections were then incubated at room temperature overnight with the following primary antibodies: Cyr61 (clone H-78; cat. no. sc-13100; dilution, 1:500; Santa Cruz Biotechnology, Inc., Dallas, TX, USA); hTRA2B [provided by Professor Stefan Stamm (27); dilution, 1:5,000]; HER4 (clone C-18, cat. no. sc-283, dilution 1:1500, Santa Cruz), CD44 (clone F10-44-2; dilution, 1:4,000; Abcam, cat. no. ab6124, Cambridge, UK); CD44v5 (clone VFF-8; dilution, 1:1,200; Abcam, cat. no. ab34235) and CD44v6 (clone VFF-7; dilution, 1:1,200; Abcam, cat. no 36). Subsequently, the primary antibodies were detected using the ImmPRESS ${ }^{\mathrm{TM}}$ HRP Universal Antibody (Anti-Mouse IgG/Anti-Rabbit IgG, Peroxidase) Polymer Detection kit (cat. no. MP-7500; Vector Laboratories, Ltd., Peterborough, U.K.), according to the manufacturer's protocol. Protein bands were then visualized using the ImmPACT DAB Peroxidase (HRP) Substrate (cat. no. SK-4105, Vector Laboratories, Ltd.) for $10 \mathrm{~min}$ at room temperature. Following 2 washing steps of 5 min each with PBS, sections were counterstained at room temperature for 10 min with Mayer's Hemalaun Solution (cat. no. 109249; Merck KGaA, Darmstadt, Germany) and dehydrated in an ascending series of alcohol concentrations. Coverslips were mounted with Entellan ${ }^{\circledR}$ New Rapid Mounting Medium (cat. no. 10796; Merck KGaA).

Assessment of Cyr61, hTRA2 $\beta, C D 44$ and HER4 expression. IHC assessment of Cyr61, hTRA2ß, CD44 (std, v5 and v6) and HER4 expression was reviewed with a Zeiss Axioplan 2 microscope (Zeiss AG, Oberkochen, Germany; magnification, x100) by an experienced pathologist. IHC staining was considered 
Table I. Clinicopathological characteristics of patients included in the present study $(n=67)$.

\begin{tabular}{|c|c|c|}
\hline Characteristics & $\begin{array}{c}\text { Number } \\
\text { of patients }\end{array}$ & $\begin{array}{l}\text { Percentage } \\
\text { of patients }\end{array}$ \\
\hline \multicolumn{3}{|l|}{ Age, years } \\
\hline$<50$ & 15 & 22.4 \\
\hline$>50$ & 52 & 77.6 \\
\hline \multicolumn{3}{|c|}{ Histological tumor type } \\
\hline Invasive ductal & 43 & 64.2 \\
\hline Invasive lobular & 12 & 17.9 \\
\hline Other & 12 & 17.9 \\
\hline \multicolumn{3}{|c|}{ Lymph node metastasis status } \\
\hline Present & 27 & 40.3 \\
\hline Absent & 40 & 59.7 \\
\hline \multicolumn{3}{|l|}{ Tumor grade } \\
\hline G1 & 2 & 3.0 \\
\hline G2 & 29 & 43.3 \\
\hline G3 & 36 & 53.7 \\
\hline \multicolumn{3}{|c|}{ ER/PR expression status } \\
\hline Positive & 49 & 73.1 \\
\hline Negative & 18 & 26.9 \\
\hline \multicolumn{3}{|c|}{ HER2 expression status } \\
\hline Score 0 & 13 & 19.4 \\
\hline Score 1 & 19 & 28.4 \\
\hline Score 2 & 9 & 13.4 \\
\hline Score 3 & 26 & 38.8 \\
\hline \multicolumn{3}{|c|}{ Molecular tumor subtype } \\
\hline Luminal like & 30 & 44.8 \\
\hline HER2/luminal & 19 & 28.4 \\
\hline HER2-like & 8 & 11.9 \\
\hline Triple negative & 10 & 14.9 \\
\hline
\end{tabular}

ER, estrogen receptor; PR, progesterone receptor; HER2, human epidermal growth factor receptor 2 .

positive if a protein signal was observed in the cytoplasm or nucleus of the BC cells. The intensity of IHC staining was classified as follows: Absent, 0; weak, 1+; moderate, 2+; and strong, 3+. A strong intensity of staining (3+) was considered to indicate overexpression (Fig. 1).

Statistical analysis. Pearson's correlation coefficients between Cyr61 expression and clinical factors, including hTRA2B, HER2, HER4, CD44std, CD44v5 and CD44v6 expression, were calculated. Multivariate logistic regression analyses were performed according to Akaike's information criterion (AIC) (28), and the model with a minimum AIC including ER/PR, CD44std and HER4 score 2 and 3 was selected. For the analysis of OS and DFS, the Kaplan-Meier estimator and Cox regression model were used. $\mathrm{P}<0.05$ was considered to indicate a statistically significant difference. All analyses were conducted using the statistical software environment $\mathrm{R}$, version 3.2.3 (29).
Table II. Multivariate regression analysis for cysteine-rich angiogenic inducer 61 with the optimal Akaike's information criterion score (86.678).

\begin{tabular}{lccc}
\hline Variable & $\begin{array}{c}\text { Regression } \\
\text { coefficient }\end{array}$ & $95 \%$ CI & P-value \\
\hline Intercept & 0.793 & $-1.323-2.908$ & 0.463 \\
ER/PR & 1.598 & $0.303-2.893$ & 0.016 \\
CD44std & -0.003 & $-0.007-0.000$ & 0.074 \\
HER4 score 2 & 0.029 & $-1.115-1.174$ & 0.960 \\
HER4 score 3 & 1.728 & $-0.743-4.199$ & 0.171 \\
\hline
\end{tabular}

CI, confidence interval; ER, estrogen receptor; PR, progesterone receptor; $\mathrm{CD}$, cluster of differentiation; HER4, human epidermal growth factor receptor 4 .

\section{Results}

Univariate analysis. Univariate analysis demonstrated a significant positive association between Cyr61 overexpression and the molecular tumor subtype of $\mathrm{BC}(\mathrm{P}=0.039$; data not shown). Cyr61 overexpression was more frequently observed in luminal-like (66.67\%) and HER2/luminal (57.89\%) molecular subtypes compared with HER2-like (12.50\%) and triple negative $(40.00 \%)$ molecular subtypes. Additionally, Cyr61 overexpression was identified to be significantly positively associated with ER/PR expression status $(\mathrm{P}=0.013$; data not shown). Cyr61 overexpression was not observed to be significantly associated with any of the other clinical factors studied.

Multivariate logistic regression analysis. The final regression model with a minimum AIC (86.678) included ER/PR expression status, CD44std, HER4 score 2 and HER4 score 3. This multivariate logistic regression analysis confirmed the significant positive association between Cyr61 overexpression and ER/PR expression status ( $\mathrm{P}=0.016$; Table II). In addition, a notable negative association was identified between Cyr61 overexpression and CD44std expression was observed $(\mathrm{P}=0.074$; Table II).

\section{Survival analysis}

Overall survival (OS). The median follow-up time was 115 months (range, 0-131 months; data not shown). During follow-up, 18 recurrences and 22 mortalities were observed (data not shown). At 60 months, 10 patients had succumbed, resulting in a 5-year OS-rate of $84.8 \%$ (Table III). At 120 months, 21 mortalities had occurred, resulting in a 10-year OS rate of $66.8 \%$ and 45 patients were censored (Table III).

OS stratified for Cyr61 expression. A subgroup analysis according to Cyr61 expression level was performed (Table III). Kaplan-Meier-survival analysis demonstrated a lower OS rate in patients with Cyr61 overexpression $(n=35)$ at 60 months (5-year OS, 82.9 vs. 87.1\%) and 120 months (10-year OS, 62.9 vs. $69.7 \%$ ) compared with patients without Cyr61 overexpression $(n=31)$. The most distinct difference between the two groups was observed at 108 months (9 years) with an OS rate of $62.9 \%$ 
Table III. OS of patients with breast cancer stratified by Cyr61 expression.

A, All patients with $\mathrm{BC}(\mathrm{n}=67)$

\begin{tabular}{|c|c|c|c|c|}
\hline Time point & Cyr61 expression level & Number of patients & Events (Mortality) & OS $(\%)$ \\
\hline \multirow[t]{3}{*}{ Baseline } & All & 66 & 0 & 100.0 \\
\hline & Scores $1+2$ & 31 & 0 & 100.0 \\
\hline & Score 3 & 35 & 0 & 100.0 \\
\hline \multirow[t]{3}{*}{60 months } & All & 57 & 10 & 84.8 \\
\hline & Scores $1+2$ & 28 & 4 & 87.1 \\
\hline & Score 3 & 30 & 6 & 82.9 \\
\hline \multirow[t]{3}{*}{108 months } & All & 47 & 20 & 69.7 \\
\hline & Scores $1+2$ & 25 & 7 & 77.4 \\
\hline & Score 3 & 23 & 13 & 62.9 \\
\hline \multirow[t]{3}{*}{120 months } & All & 24 & 21 & 66.8 \\
\hline & Scores $1+2$ & 10 & 8 & 69.7 \\
\hline & Score 3 & 23 & 13 & 62.9 \\
\hline
\end{tabular}

B, Patients with HR-positive BC ( $\mathrm{n}=49)$

\begin{tabular}{|c|c|c|c|c|}
\hline Time point & Cyr61 expression level & Number of patients & Events & OS $(\%)$ \\
\hline \multirow[t]{3}{*}{ Baseline } & All & 48 & 0 & 100.0 \\
\hline & Scores $1+2$ & 18 & 0 & 100.0 \\
\hline & Score 3 & 30 & 0 & 100.0 \\
\hline \multirow[t]{3}{*}{60 months } & All & 42 & 7 & 85.4 \\
\hline & Scores $1+2$ & 17 & 2 & 88.9 \\
\hline & Score 3 & 26 & 5 & 83.3 \\
\hline \multirow[t]{3}{*}{108 months } & All & 33 & 16 & 66.7 \\
\hline & Scores $1+2$ & 15 & 4 & 77.8 \\
\hline & Score 3 & 19 & 12 & 60.0 \\
\hline \multirow[t]{3}{*}{120 months } & All & 17 & 17 & 62.7 \\
\hline & Scores $1+2$ & 5 & 5 & 62.2 \\
\hline & Score 3 & 19 & 12 & 60.0 \\
\hline
\end{tabular}

A total of 1 patient was deleted in both analyses at baseline due to missing follow-up data. OS, overall survival; Cyr61, cysteine-rich angiogenic inducer 61 .
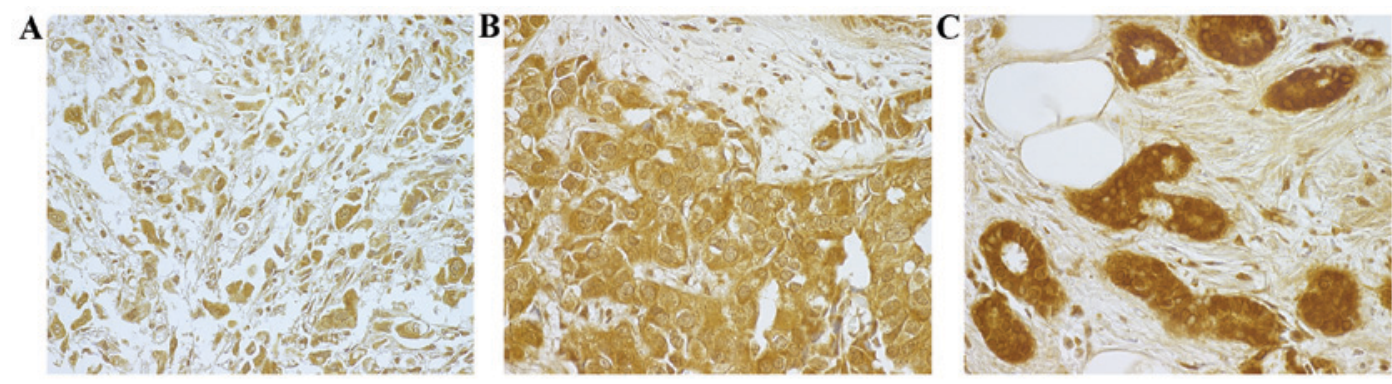

Figure 1. Immunohistochemical analysis of Cyr61 protein expression in primary non-metastatic breast cancer sections. Specific nuclear and cytoplasmic expression of Cyr61 in invasive carcinomas of the breast were observed following staining with hemalum. (A) Weak expression, score 1+; (B) moderate expression, score 2+; and (C) strong expression, score 3+. Magnification, x400. Cyr61, cysteine-rich angiogenic inducer 61.

in patients with Cyr61-overexpression and $77.4 \%$ in patients without Cyr61 overexpression (Table III). However, using the
Cox regression model, a significant association between Cyr61 overexpression and OS was not detected ( $\mathrm{P}=0.519$; Fig. 2). 
Table IV. PFS of patients with breast cancer stratified by Cyr61 expression.

A, All patients with $\mathrm{BC}(\mathrm{n}=67)$

\begin{tabular}{|c|c|c|c|c|}
\hline Time point & Cyr61 expression level & Number of patients & $\begin{array}{c}\text { Events (Mortalities } \\
\text { and recurrence) }\end{array}$ & PFS (\%) \\
\hline \multirow[t]{3}{*}{ Baseline } & All & 66 & 0 & 100.0 \\
\hline & Score $1+2$ & 31 & 0 & 100.0 \\
\hline & Score 3 & 35 & 0 & 100.0 \\
\hline \multirow[t]{3}{*}{60 months } & All & 48 & 20 & 69.7 \\
\hline & Score $1+2$ & 25 & 8 & 74.2 \\
\hline & Score 3 & 24 & 12 & 65.7 \\
\hline \multirow[t]{3}{*}{108 months } & All & 40 & 25 & 62.0 \\
\hline & Score $1+2$ & 20 & 11 & 64.4 \\
\hline & Score 3 & 22 & 14 & 60.0 \\
\hline \multirow[t]{3}{*}{120 months } & All & 23 & 27 & 57.0 \\
\hline & Score $1+2$ & 9 & 12 & 57.2 \\
\hline & Score 3 & 15 & 15 & 56.0 \\
\hline
\end{tabular}

B, Patients with HR-positive BC $(n=49)$

\begin{tabular}{|c|c|c|c|c|}
\hline Time point & Cyr61 expression level & Number of patients & Events & PFS $(\%)$ \\
\hline \multirow[t]{3}{*}{ Baseline } & All & 48 & 0 & 100.0 \\
\hline & Scores $1+2$ & 18 & 0 & 100.0 \\
\hline & Score 3 & 30 & 0 & 100.0 \\
\hline \multirow[t]{3}{*}{60 months } & All & 36 & 14 & 70.8 \\
\hline & Scores $1+2$ & 16 & 4 & 77.8 \\
\hline & Score 3 & 21 & 10 & 66.7 \\
\hline \multirow[t]{3}{*}{108 months } & All & 29 & 18 & 62.4 \\
\hline & Scores $1+2$ & 12 & 6 & 66.2 \\
\hline & Score 3 & 19 & 12 & 60.0 \\
\hline \multirow[t]{3}{*}{120 months } & All & 17 & 20 & 55.6 \\
\hline & Scores $1+2$ & 5 & 7 & 53.0 \\
\hline & Score 3 & 19 & 12 & 60.0 \\
\hline
\end{tabular}

A total of 1 patient was deleted in both analyses at baseline due to missing follow-up data. PFS, progression free survival; Cyr61, cysteine-rich angiogenic inducer 61 .

OS stratified for HR status and Cyr61 expression. The OS subgroup analysis for patients with HR-positive BC $(n=48)$ demonstrated a 5-year OS rate of $85.4 \%$, a 9-year OS rate of $66.7 \%$ and a 10 -year OS rate of $62.7 \%$ (Table III). Stratifying this subgroup according to Cyr61 expression also revealed a lower OS rates for patients with Cyr61 overexpression compared with patients without Cyr61 overexpression at 60 months ( 83.3 vs. $88.9 \%$ ) and at 108 months (60.0 vs. $77.8 \%)$, but not at 120 months (60.0 vs. 62.2\%) (Table III). However, a significant difference between these two groups was not observed based on the Cox regression model $(\mathrm{P}=0.397$; data not shown).

Progression free survival (PFS). During follow-up, 17 recurrences were observed (0-60 months, 14 recurrences;
61-131 months, 3 recurrences) resulting in a recurrence rate of $25.4 \%$ (data not shown). A total of 12/17 (70.6\%) patients with a recurrence succumbed during this time (data not shown). During follow-up for PFS, a total of 27 events (e.g. mortalities plus recurrences) were observed and 39 patients were censored (Table IV).

PFS stratified for Cyr61 expression. Compared with patients without Cyr61 overexpression, patients with Cyr61 overexpression were more likely to experience a recurrence (31.4 vs. $19.4 \%$ ) and exhibited a higher risk of mortality subsequent to recurrence ( 81.8 vs. $50.0 \%$ ) (data not shown). Kaplan-Meier estimator analysis demonstrated a lower PFS for patients with Cyr61 overexpression compared with those without Cyr61 overexpression at 60 months (65.7 vs. $74.2 \%)$, whereas at 


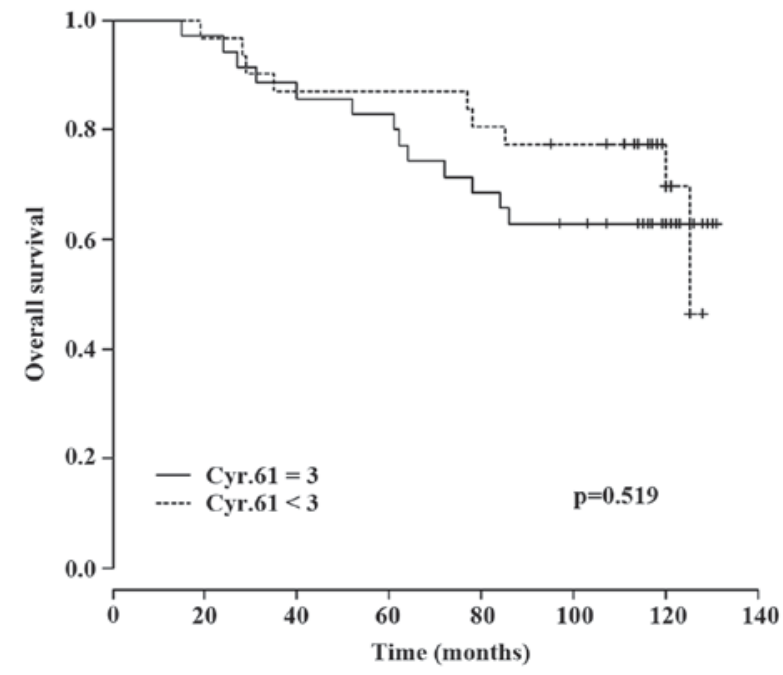

Figure 2. Overall survival of patients with primary non-metastatic breast cancer in regards to Cyr61 expression levels. Kaplan-Meier estimator curves stratified for Cyr61 expression. Cyr61, cysteine-rich angiogenic inducer 61.

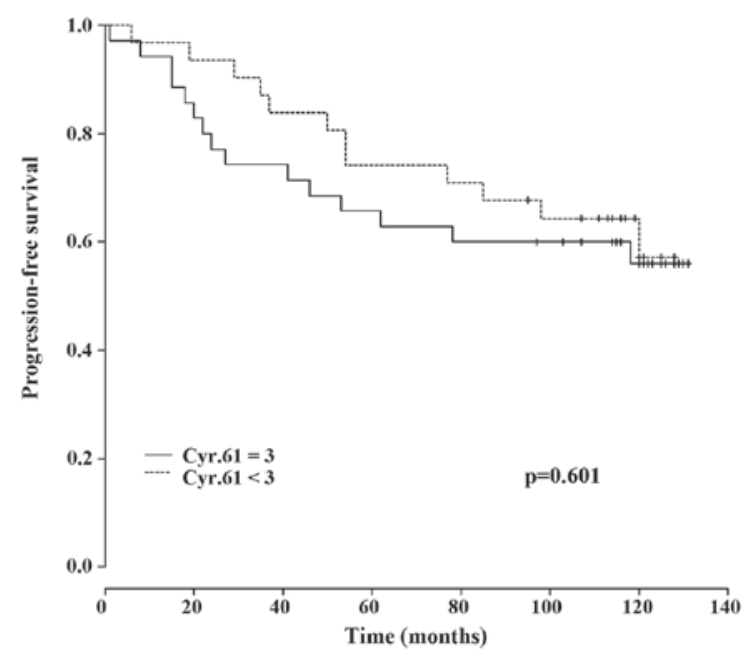

Figure 3. Progression-free survival patients with primary non-metastatic breast cancer in regards to Cyr61 expression levels. Kaplan-Meier estimator curves stratified for Cyr61 expression. Cyr61, cysteine-rich angiogenic inducer 61 .

120 months (56.0 vs. 57.2\%) no notable difference in PFS was observed between the two groups (Table IV). Therefore, no significant association between Cyr61 overexpression and PFS was detected ( $\mathrm{P}=0.601$; Fig. 3).

PFS stratified for HR status and Cyr61 expression. Subgroup analysis for PFS in regard to HR status also revealed a higher recurrence rate for patients with Cyr61 overexpression compared with patients without Cyr61 overexpression (33.3 vs. $16.7 \%)$, in addition to a higher mortality rate (90.0 vs. $33.3 \%$ ) (data not shown). Kaplan-Meier estimator analysis in regard to HR status (HR positive vs. HR negative expression) demonstrated a lower PFS for HR positive patients with Cyr61 overexpression compared with patients without Cyr61 overexpression at 60 months (66.7 vs. $77.8 \%)$, whereas at 120 months (55.4 vs. $53.0 \%$ ) no marked difference was observed between the two groups (Table IV). Thus, no significant association between Cyr61 overexpression and PFS in HR positive patients was demonstrated ( $\mathrm{P}=0.638$; data not shown).

\section{Discussion}

In the present study, based on 67 patients with primary $\mathrm{BC}$, a positive association between Cyr61 expression and ER/PR expression status was observed. This data was consistent in univariate and multivariate analyses. In addition, an association between Cyr61 overexpression and the BC molecular subtype was observed, with increased Cyr61 overexpression rates in luminal-like and HER2/luminal tumors. In multivariate logistic regression analysis, a notable negative association between Cyr61 and CD44std expression was observed.

These data are supported by the patient outcome data, which revealed increased recurrence rates and decreased OS rates in patients with Cyr61 overexpression compared with those without Cyr61 overexpression. In particular, long-term OS was impaired in patients with Cyr61 overexpression. Similar results were obtained in patients with ER/PR positive BC. However, the observed OS trend did not reach statistical significance. Potential reasons for this include the relatively small patient cohort and the high number of censored patients at the end of the observation.

Resistance to endocrine therapy is a major challenge in the treatment of patients with BC. Although a range of signaling pathways have been identified to serve a role in this treatment resistance, the highly complex and heterogeneous underlying molecular mechanisms are not completely understood. The results of the present study support the findings of a previous study by Tsai et al (24), which reported an association between Cyr61 and carcinogenesis, tumor invasiveness, and the induction of estrogen-independence and anti-estrogen resistance. These data are in accordance with the data of a study by Jia et al (21), which reported that Cyr61 contributes to the poor response to letrozole treatment in patients with ER positive BC.

The results of these previous studies and the present study indicate that Cyr61 serves an important role in the development of endocrine treatment resistance in BC. Although the results for the association between survival and Cyr61 overexpression did not reach a statistically significant level in the present study, they do suggest that patients with BC overexpressing Cyr61 exhibit a decreased long-term survival. In order to confirm the clinical significance of Cyr61 as a marker for OS and PFS, additional long-term survival analyses with larger patient populations are required.

In conclusion, the results of the present study indicate that Cyr61 overexpression serves a role in the development of endocrine treatment resistance in patients with $\mathrm{BC}$. In addition, the results of the present study identify Cyr61 as a potential therapeutic target to overcome endocrine therapy resistance.

\section{References}

1. Johnston SR and Dowsett M: Aromatase inhibitors for breast cancer: Lessons from the laboratory. Nat Rev Cancer 3: 821-831, 2003.

2. Massarweh S and Schiff R: Resistance to endocrine therapy in breast cancer: Exploiting estrogen receptor/growth factor signaling crosstalk. Endocr Relat Cancer 13 (Suppl 1): S15-S24, 2006. 
3. Early Breast Cancer Trialists' Collaborative Group (EBCTCG) Davies C, Godwin J, Gray R, Clarke M, Cutter D, Darby S, McGale P, Pan HC, Taylor C, et al: Relevance of breast cancer hormone receptors and other factors to the efficacy of adjuvant tamoxifen: Patient-level meta-analysis of randomised trials Lancet 378: 771-784, 2011.

4. García-Becerra R, Santos N, Díaz L and Camacho J: Mechanisms of resistance to endocrine therapy in breast cancer: Focus on signaling pathways, miRNAs and genetically based resistance. Int J Mol Sci 14: 108-145, 2012.

5. Rugo HS, Vidula $\mathrm{N}$ and Ma C: Improving response to hormone therapy in breast cancer: New targets, new therapeutic options Am Soc Clin Oncol Educ Book 35: e40-e54, 2016.

6. Lau LF: CCN1/CYR61: The very model of a modern matricellular protein. Cell Mol Life Sci 68: 3149-3163, 2011.

7. Chen Y and Du XY: Functional properties and intracellular signaling of CCN1/Cyr61. J Cell Biochem 100: 1337-1345, 2007.

8. Katsube K, Sakamoto K, Tamamura Y and Yamaguchi A: Role of CCN, a vertebrate specific gene family, in development. Dev Growth Differ 51: 55-67, 2009.

9. Leask A and Abraham DJ: All in the CCN family: Essential matricellular signaling modulators emerge from the bunker. J Cell Sci 119: 4803-4810, 2006.

10. Lau LF and Lam SC: The CCN family of angiogenic regulators: The integrin connection. Exp Cell Res 248: 44-57, 1999.

11. Emre Y and Imhof BA: Matricellular protein CCN1/CYR61: A new player in inflammation and leukocyte trafficking. Semin Immunopathol 36: 253-259, 2014.

12. Weiskirchen R and Tacke F: Liver Fibrosis: From pathogenesis to novel therapies. Dig Dis 34: 410-422, 2016.

13. Xu T, He YH, Wang MQ, Yao HW, Ni MM, Zhang L, Meng XM Huang C, Ge YX and Li J: Therapeutic potential of cysteine-rich protein 61 in rheumatoid arthritis. Gene 592: 179-185, 2016.

14. Li J, Ye L, Owen S, Weeks HP, Zhang Z and Jiang WG: Emerging role of CCN family proteins in tumorigenesis and cancer metastasis (Review). Int J Mol Med 36: 1451-1463, 2015.

15. Yeger H and Perbal B: CCN family of proteins: Critical modulators of the tumor cell microenvironment. J Cell Commun Signal 10: 229-240, 2016

16. Hirschfeld M, Jaeger M, Buratti E, Stuani C, Grueneisen J, Gitsch G and Stickeler E: Expression of tumor-promoting Cyr61 is regulated by hTRA2- $\beta 1$ and acidosis. Hum Mol Genet 20 2356-2365, 2011.

17. Hirschfeld M, zur Hausen A, Bettendorf H, Jäger M and Stickeler E: Alternative splicing of Cyr61 is regulated by hypoxia and significantly changed in breast cancer. Cancer Res 69: 2082-2090, 2009.
18. Sánchez-Bailón MP, Calcabrini A, Mayoral-Varo V, Molinari A, Wagner KU, Losada JP, Ciordia S, Albar JP and Martín-Pérez J: Cyr61 as mediator of Src signaling in triple negative breast cancer cells. Oncotarget 6: 13520-13538, 2015.

19. Sarkissyan S, Sarkissyan M, Wu Y, Cardenas J, Koeffler HP and Vadgama JV: IGF-1 regulates Cyr61 induced breast cancer cell proliferation and invasion. PloS One 9: e103534, 2014.

20. Huber MC, Falkenberg N, Hauck SM, Priller M, Braselmann H, Feuchtinger A, Walch A, Schmitt M and Aubele M: Cyr61 and YB-1 are novel interacting partners of uPAR and elevate the malignancy of triple-negative breast cancer. Oncotarget 7: 44062-44075, 2016.

21. Jia X, Liu G, Cheng J, Shen Z and Shao Z: CYR61 contributes to poor response to letrozole in ER positive breast carcinoma. Curr Cancer Drug Targets, 2016.

22. Lin J, Huo R, Wang L, Zhou Z, Sun Y, Shen B, Wang R and Li N: A novel anti-Cyr61 antibody inhibits breast cancer growth and metastasis in vivo. Cancer Immunol Immunother 61: 677-687, 2012.

23. Saglam O, Dai F, Husain S, Zhan Y, Toruner G and Haines GK III: Matricellular protein CCN1 (CYR61) expression is associated with high-grade ductal carcinoma in situ. Hum Pathol 45: 1269-1275, 2014

24. Tsai MS, Bogart DF, Castañeda JM, Li P and Lupu R: Cyr61 promotes breast tumorigenesis and cancer progression. Oncogene 21: 8178-8185, 2002.

25. The world Health Organization Histological Typing of Breast Tumors-Second Edition. The World Organization. Am J Clin Pathol 78: 806-816, 1982

26. Harbeck N and Rody A: Diagnostik und Therapie primärer metastasierter Mammakarzinome http://www.ago-online. $\mathrm{de} /$ fileadmin/downloads/leitlinien/mamma/2017-03/AGO_ deutsch/PDF Einzeldateien deutsch/2017D\%2005 Prognostische\%20 und\%20praediktive\%20Faktoren.pdf. AGO in e.V. DGGG e.V., DKG e.V.2017.

27. Stoilov P, Daoud R, Nayler O and Stamm S: Human tra2-beta1 autoregulates its protein concentration by influencing alternative splicing of its pre-mRNA. Hum Mol Genet 13: 509-524, 2004

28. Schwarz G: Estimating the dimensions of a model. Ann Stat 6: 461-464, 1978

29. R Core Team (2016). R: A language and environment for statistical computing. R Foundation for Statistical Computing, Vienna, Austria. https://www.R-project.org/. Accessed May 25, 2016. 